\title{
Solvability of Discrete Two-point Boundary Value Problems
}

\author{
M. Mohamed (Corresponding author) \\ Jabatan Matematik \& Statistik \\ Universiti Teknologi MARA (UiTM) Malaysia \\ 02600 Arau, Perlis, Malaysia \\ E-mail: mesliza@perlis.uitm.edu.my \\ H.B. Thompson \\ Department of Mathematics, The University of Queensland \\ Queensland 4072, Australia \\ E-mail: hbt@maths.uq.edu.au \\ M. S. Jusoh \\ Fakulti Kejuruteraan Awam \\ Universiti Teknologi MARA (UiTM) Malaysia \\ 02600 Arau, Perlis, Malaysia \\ E-mail: muhdpian@yahoo.com
}

\begin{abstract}
We study the discrete approximation to solutions of first-order system arising from applying the trapezoidal rule to a second-order scalar ordinary differential equation. In the trapezoidal rule the finite difference approximation are $D y_{k}=$ $\left(z_{k}+z_{k-1}\right) / 2, D z_{k}=\left(f_{k}+f_{k-1}\right) / 2$, for $k=1,2, . ., n$, and $t_{k}=k h$ for $\left.k=0, \ldots, n, 0=G\left(\left(y_{0}, y_{n}\right),\left(z_{0}+z_{1}\right) / 2,\left(z_{n-1}+z_{n}\right) / 2\right)\right)$, where $f_{i} \equiv f\left(t_{i}, y_{i}, z_{i}\right)$ and $G=\left(g^{0}, g^{1}\right)$ are continuous and fully nonlinear. We assume there exist strict discrete lower and strict discrete upper solutions and impose additional conditions on $f_{k}$ and $G$ which are known to yield a priori bounds on, and to guarantee the existence of solutions of the discrete approximation for sufficiently small grid size. We use the homotopy to compute the solutions of the discrete approximations. In this paper we study the first-order system of difference equations that arise when one applies the trapezoidal rule to approximate solutions of the second-order scalar ordinary differential equation.
\end{abstract}

Keywords: Second order scalar ordinary differential equation, Strict discrete lower and strict discrete upper solutions, Bernstein-Nagumo condition, Brouwer degree theory

\section{Introduction}

Consider the continuous two-point boundary value problem

$$
\begin{array}{r}
y^{\prime \prime}=f\left(t, y, y^{\prime}\right), t \in[0,1], \\
G\left((y(0), y(1)),\left(y^{\prime}(0), y^{\prime}(1)\right)\right)=0
\end{array}
$$

where $f:[0,1] \times \mathbb{R}^{2} \rightarrow \mathbb{R}$ and $G=\left(g^{0}, g^{1}\right), g^{i}: \mathbb{R}^{2} \times \mathbb{R}^{2} \rightarrow \mathbb{R}, i=0,1$ are continuous and fully nonlinear. The trapezoidal rule for solving (1), (2) involves rewriting the problem as the first-order system (see for example Russell,1977 and Denny and Landis, 1972),

$$
\begin{aligned}
y^{\prime} & =z \\
z^{\prime} & =f(t, y, z) .
\end{aligned}
$$

Its discretization has the form

$$
\begin{array}{r}
D y_{k}=\left(z_{k}+z_{k-1}\right) / 2=: r_{k}(\mathbf{y}, \mathbf{z}), \\
D z_{k}=\left(f_{k}+f_{k-1}\right) / 2=: p_{k}(\mathbf{y}, \mathbf{z}), \\
G\left(\left(y_{0}, y_{n}\right),\left(\left(z_{0}+z_{1}\right) / 2,\left(z_{n-1}+z_{n}\right) / 2\right)\right)=0,
\end{array}
$$

where the grid size $h=1 / n, D y_{k}=\left(y_{k}-y_{k-1}\right) / h, D z_{k}=\left(z_{k}-z_{k-1}\right) / h$ for $k=1,2, \cdots, n$ and the grid points $t_{k}=k h$ for $k=0,1, \cdots, n$, and $f_{i} \equiv f\left(t_{i}, y_{i}, z_{i}\right)$. 
If $y=y(t)$ is a solution of (1), then $y=y(t)$ and $z=y^{\prime}(t)$ is a solution of the system (3), (4). By a solution of (5), (6) and (7), we mean a vector $\mathbf{y}=\left(y_{0}, y_{1}, \cdots, y_{n}\right)$ and $\mathbf{z}=\left(z_{0}, z_{1}, \cdots, z_{n}\right)$ satisfying (5) and (6) for $k=1,2, \cdots, n$ and (7).

Replacing $k$ by $k+1$ in (6), we get

$$
D z_{k+1}=\left(f_{k+1}+f_{k}\right) / 2
$$

Hence a solution of the discrete approximation, (5) and (6) is a solution of the second order difference equation

$$
D^{2} y_{k+1}=D\left(D y_{k+1}\right)=D\left(z_{k+1}+z_{k}\right) / 2=\left(f_{k-1}+2 f_{k}+f_{k+1}\right) / 4=: g_{k}(\mathbf{y}, \mathbf{z})
$$

where $f_{j} \equiv f\left(t_{j}, y_{j}, z_{j}\right)$ for $j=k-1, k, k+1$ and $k=1, \cdots, n-1$.

Following Henderson and Thompson (2001) we assume that there are strict lower and strict upper solutions for (1), $\alpha$ and $\beta$, respectively, which are very strongly compatible with the very general nonlinear boundary conditions given by $G=0$. Moreover we assume that the right hand side of (1), $f$, satisfies a Nagumo growth bound with respect to $y^{\prime}$ for $(t, y)$ satisfying $\alpha(t) \leq y \leq \beta(t)$.

Thompson (1996) introduced the notion of compatibility of boundary conditions with lower and upper solutions and established the compatibility conditions for the Sturm-Liouville, the periodic, and the Neumann boundary conditions. Moreover he showed that under the above assumptions there are solutions of (1) and (2).

Henderson and Thompson (2001) showed that under these assumptions $\boldsymbol{\alpha}=\left(\alpha\left(t_{0}\right), \cdots, \alpha\left(t_{n}\right)\right)$ and $\boldsymbol{\beta}=\left(\beta\left(t_{0}\right), \cdots, \beta\left(t_{n}\right)\right)$ are strict discrete lower and strict discrete upper solutions, respectively, for (9) provided the step size $h=t_{i}-t_{i-1}=1 / n$ is sufficiently small. Using these strict discrete lower and strict discrete upper solutions and the Nagumo growth bound they established a priori bounds on difference quotients of solutions independently of step size provided the step size is sufficiently small. They introduced the central notion of very strong discrete compatibility of the boundary conditions $G=0$ with the strict discrete lower and strict discrete upper solutions, $\boldsymbol{\alpha}$ and $\boldsymbol{\beta}$, respectively. Under the assumptions of Henderson and Thompson (2001), they showed the boundary conditions are effectively very strong discrete compatible with $\boldsymbol{\alpha}$ and $\boldsymbol{\beta}$ and exploited this to show that solutions of the discrete problem exist for sufficiently small step size.

We introduce a variant of strict discrete lower and strict discrete upper solutions $\boldsymbol{\alpha}$ and $\boldsymbol{\beta}$, respectively, appropriate for the study of (9). We show that $\boldsymbol{\alpha}=\left(\alpha\left(t_{0}\right), \cdots, \alpha\left(t_{n}\right)\right)$, and $\boldsymbol{\beta}=\left(\beta\left(t_{0}\right), \cdots, \beta\left(t_{n}\right)\right)$ are strict discrete lower and strict discrete upper solutions, respectively, for (9) provided the step size is sufficiently small. Combining these with the Nagumo growth bound we establish a priori bounds on the $z_{k}$ and hence on $D y_{k}$ for solutions of (5) and (6) independent of the step size for sufficiently small step size.

We introduce the central notion of very strong discrete compatibility of the boundary conditions $G=0$ with $\boldsymbol{\alpha}$ and $\boldsymbol{\beta}$. Following Henderson and Thompson we show that our boundary conditions (6) are effectively very strongly discrete compatible with $\boldsymbol{\alpha}$ and $\boldsymbol{\beta}$. Our boundary conditions (7) are a variant of those of Henderson and Thompson and again we need to modify Henderson and Thompson's arguments. We use this machinery to show that solutions of our discrete problem exist for sufficiently small step size.

In order to state our results we need some notation. We denote the boundary of a set $T$ by $\partial T$ and the closure of $T$ by $\bar{T}$. As usual $C^{m}(A ; B)$ denotes the space of $\mathrm{m}$ times continuously differentiable functions from $\mathrm{A}$ to $\mathrm{B}$ endowed with the maximum norm. In the case of continuous functions we abbreviate this to $C(A ; B)$. In the case $B=\mathbb{R}$ we omit the $B$. For any vector $\mathbf{s}=\left(s_{0}, \cdots, s_{n}\right) \in \mathbb{R}^{n+1}$ and $\mathbf{u}=\left(u_{0}, \cdots, u_{n}\right) \in \mathbb{R}^{n+1}$ we write $\mathbf{s} \leq \mathbf{u}$ if $s_{k} \leq u_{k}$ for $k=0, \cdots, n$. If $\mathbf{y}=\left(y_{0}, \cdots, y_{n}\right) \in \mathbb{R}^{n+1}$, then set $D y_{k}=\left(y_{k}-y_{k-1}\right) / h$ for $1 \leq k \leq n$. If $l:[0,1] \rightarrow \mathbb{R}$ and $n>0$ is an integer, then we set $\mathbf{l}=\left(l_{0}, \cdots, l_{n}\right) \in \mathbb{R}^{n+1}$, where $l_{k}=l(k / n)$ for $k=0, \cdots, n$. If $c \in \mathbb{R}$ is a constant then set $\mathbf{c}=\left(c_{0}, \ldots, c_{n}\right)$, where $c_{k}=c$ for all $k$.

If $\mathrm{A}$ is a bounded open subset of $\mathbb{R}^{n}, p \in \mathbb{R}^{n}, f \in C\left(\bar{A} ; \mathbb{R}^{n}\right)$ and $p \notin f(\partial A)$ we denote the Brouwer degree of $f$ at $p$ by $d(f, A, p)$.

\section{Preliminary Results}

Definition 1. We call $\alpha(\beta)$ a strict lower (strict upper) solution for (1) if $\alpha(\beta) \in C^{2}[0,1]$, and there exists $\gamma>0$ such that

$$
\begin{gathered}
\alpha^{\prime \prime}(t)-f\left(t, \alpha(t), \alpha^{\prime}(t)\right) \geq \gamma, t \in[0,1] \\
\left.\left(f\left(t, \beta(t), \beta^{\prime}(t)\right)-\beta^{\prime \prime}(t)\right) \geq \gamma, t \in[0,1]\right) .
\end{gathered}
$$

We will call the pair non-degenerate if $\Delta=(\alpha(0), \beta(0)) \times(\alpha(1), \beta(1))$ is non-empty. If $\gamma=0$, in (10), (11) then we call $\alpha$ ( $\beta$ ) a lower (upper) solution for (1). 
We call $\boldsymbol{\alpha}(\boldsymbol{\beta})$ a strict discrete lower (strict discrete upper) solution for (9) if there is $\gamma>0$ such that

$$
\begin{gathered}
D^{2} \alpha_{k+1}-\frac{f\left(t_{k+1}, \alpha_{k+1}, D \alpha_{k+1}\right)}{4}-\frac{2 f\left(t_{k}, \alpha_{k}, D \alpha_{k}\right)}{4}-\frac{f\left(t_{k-1}, \alpha_{k-1}, D \alpha_{k-1}\right)}{4} \geq \gamma, \\
\left(\frac{f\left(t_{k+1}, \beta_{k+1}, D \beta_{k+1}\right)}{4}+\frac{2 f\left(t_{k}, \beta_{k}, D \beta_{k}\right)}{4}+\frac{f\left(t_{k-1}, \beta_{k-1}, D \beta_{k-1}\right)}{4}\right. \\
\left.-D^{2} \beta_{k+1} \geq \gamma\right)
\end{gathered}
$$

for $k=1, \cdots, n-1$ where

$$
\begin{aligned}
& \alpha_{k}=\alpha\left(t_{k}\right), \boldsymbol{\alpha}=\left(\alpha_{0}, \alpha_{1}, \cdots, \alpha_{n}\right), \\
& \beta_{k}=\beta\left(t_{k}\right), \boldsymbol{\beta}=\left(\beta_{0}, \beta_{1}, \cdots, \beta_{n}\right) .
\end{aligned}
$$

If $\gamma=0$, then we call $\boldsymbol{\alpha}(\boldsymbol{\beta})$ a discrete lower (discrete upper) solution for (9). In view of (10) and (11) by abuse of notation, we set $\Delta=\left(\alpha_{0}, \beta_{0}\right) \times\left(\alpha_{n}, \beta_{n}\right)$ and say $\boldsymbol{\alpha}$ and $\boldsymbol{\beta}$ are nondegenerate if $\Delta$ is nonempty.

To state our main result we will need the following variant of the Bernstein-Nagumo condition.

Definition 2. Let $\alpha \leq y \leq \beta$ be strict lower and strict upper solutions for (1). We say the $f$ satisfies the Bernstein-Nagumo condition for if there exists nondecreasing $\phi \in C([0, \infty] ;(0, \infty))$ and $N>0$ such that

$$
|f(t, y, p)| \leq \phi(|p|) \text { for all } t \in[0,1] \text { and } y \in[\alpha(t), \beta(t)]
$$

and

$$
\int_{\sigma}^{N} \frac{s d s}{h(s)}>\max \{\beta(t): t \in[0,1]\}-\min \{\alpha(t): t \in[0,1]\}
$$

where $\sigma=\max \{|\beta(1)-\alpha(0)|,|\beta(0)-\alpha(1)|\}$.

As indicated earlier, we use the strict discrete lower and strict discrete upper solutions with maximum principle arguments to obtain a priori bounds on solutions of the discrete problem. Moreover we use the following discrete version of the Bernstein-Nagumo condition to obtain a priori bounds on $z_{k}$ for solutions of the discrete problem.

Definition 3. (see Henderson and Thompson, 2001) Let $\boldsymbol{\alpha} \leq \mathbf{y} \leq \boldsymbol{\beta}$ be strict discrete lower and strict discrete upper solutions for (9). We say the $f_{k}$ satisfy the discrete Bernstein-Nagumo condition for $k=1, \cdots, n$ if there exists nondecreasing $\phi \in C([0, \infty] ;(0, \infty))$ and $N>0$ such that

$$
\left|f\left(t_{k}, y_{k}, p\right)\right| \leq \phi(|p|) \text { for all } y_{k} \in\left[\alpha_{k}, \beta_{k}\right], k=1,2, \cdots, n
$$

and

$$
\int_{\sigma}^{N} \frac{s d s}{h(s)}>\max \left\{\beta_{k}: k=0, \cdots, n\right\}-\min \left\{\alpha_{k}: k=0, \cdots, n\right\}
$$

where $\sigma=\max \left\{\left|\beta_{n}-\alpha_{0}\right|,\left|\beta_{0}-\alpha_{n}\right|\right\}$.

Note that if $\alpha$ and $\beta$ are strict lower and strict upper solutions for (1) and $f(t, y, p)$ satisfies the Bernstein Nagumo condition with respect to $\alpha$ and $\beta$ then the $f_{k}$ satisfy the discrete Bernstein Nagumo condition with respect to $\boldsymbol{\alpha}$ and $\boldsymbol{\beta}$ given by (14) and (15).

Existence proofs for BVPs commonly employ modifications on $f$. We will make the necessary modifications by using the following notation.

If $c \leq d$ are given, let $\pi: \mathbb{R} \rightarrow[c, d]$ be the retraction given by

$$
\pi(y, c, d)=\max \{\min \{d, y\}, c\} .
$$

For each $\epsilon>0$, let $K \in C(\mathbb{R} \times(0, \infty) ;[-1,1])$ satisfy
(1) $K(., \epsilon)$ is an odd function
(2) $K(t, \epsilon)=0$, iff $t=0$ and
(3) $K(t, \epsilon)=1$ for all $t \geq \epsilon$. 
If $c \leq d$ and $\epsilon>0$ are given, let $T \in C(\mathbb{R})$ be given by

$$
T(y, c, d, \epsilon)=K(y-\pi(y, c, d), \epsilon) .
$$

Let

$$
\begin{aligned}
\tilde{f}_{k} & =f\left(t_{k}, \pi\left(y_{k}, \alpha_{k}, \beta_{k}\right), \pi(p,-L, L)\right), \text { and } \\
D^{2} y_{k+1}= & \left(1-\left|T\left(y_{k}, \alpha_{k}, \beta_{k}, \epsilon\right)\right|\right)\left(\tilde{f}_{k+1}+2 \tilde{f}_{k}+\tilde{f}_{k-1}\right) / 4+ \\
& \left.T\left(y_{k}, \alpha_{k}, \beta_{k}, \epsilon\right)\left(\mid \tilde{f}_{k+1}+2 \tilde{f}_{k}+\tilde{f}_{k-1}\right) / 4 \mid+\epsilon\right) .
\end{aligned}
$$

Let $Q:[0,1] \times[0,1] \rightarrow \mathbb{R}$ be given by

$$
Q(x, t)= \begin{cases}(1-x) t, & \text { for all } 0 \leq t \leq x \leq 1, \\ (1-t) x, & \text { for all } 0 \leq x \leq t \leq 1,\end{cases}
$$

and for all $C, D \in \mathbb{R}$, let $w(C, D):[0,1] \rightarrow \mathbb{R}$ be given by $w(C, D)(x)=C(1-x)+D x$. Let $\mathbf{X}=\mathbb{R}^{n+1} \times \mathbb{R}^{n+1} \times \mathbb{R}^{2}$ with the usual product norm. Define $\mathbf{T}: \mathbb{R}^{n+1} \rightarrow \mathbb{R}^{n+1}$ by

$$
\mathbf{T}(\mathbf{g})_{k}=-h \sum_{i=0}^{n} Q\left(t_{k}, t_{i}\right)\left(g_{i}\right) \quad \text { for } \quad k=0,1, \cdots, n
$$

for all $\mathbf{g} \in \mathbb{R}^{n+1}$. Clearly $\mathbf{T}$ is continuous and

$$
\begin{aligned}
D^{2} y_{k+1} & =g_{k}, \quad k=1, \cdots, n-1 \\
y_{0} & =A, \quad y_{n}=B
\end{aligned}
$$

if and only if

$$
\mathbf{y}=\mathbf{T}(\mathbf{g})+\mathbf{w}(A, B)
$$

Thus $(\mathbf{y}, \mathbf{z})$ is a solution of

$$
\begin{gathered}
D^{2} y_{k+1}=\left(f_{k+1}+2 f_{k}+f_{k-1}\right) / 4=g_{k}(\mathbf{y}, \mathbf{z}), \quad k=1, \cdots, n-1 \text { and } \\
z_{k}=\sum_{i=1}^{k} h D z_{i}+z_{0}=\sum_{i=1}^{k} h p_{i}+z_{0}=: l_{k}(\mathbf{y}, \mathbf{z}), \quad k=1, \cdots, n \\
y_{0}=A, \quad y_{n}=B \\
z_{0}=2\left(y_{1}-y_{0}\right) / h-z_{1}
\end{gathered}
$$

if and only if

$$
\left(\begin{array}{l}
\mathbf{y} \\
\mathbf{z}
\end{array}\right)=\left(\begin{array}{c}
\mathbf{T}(\mathbf{g}(\mathbf{y}, \mathbf{z}))+\mathbf{w}(A, B) \\
\mathbf{l}(\mathbf{y}, \mathbf{z})
\end{array}\right)
$$

where

$$
l_{0}(\mathbf{y}, \mathbf{z})=2\left(y_{1}-y_{0}\right) / h-z_{1} .
$$

Let $(\mathbf{y}, \mathbf{z})$ be a solution of (27). We show that it is a solution of (5) and (6).

Let $h_{k}=D y_{k}-\left(z_{k}+z_{k-1}\right) / 2$. Then

$$
\begin{aligned}
D h_{k} & =D^{2} y_{k}-\left(D z_{k}+D z_{k-1}\right) / 2 \\
& =g_{k}-\left(p_{k}+p_{k-1}\right) / 2 \\
& =0, \text { for } k=2, \ldots, n
\end{aligned}
$$

Thus

$$
\begin{aligned}
h_{k} & =h_{1}, \quad \text { for } 1 \leq k \leq n \\
& =D y_{1}-\left(z_{1}+z_{0}\right) / 2=0 .
\end{aligned}
$$

Thus $(\mathbf{y}, \mathbf{z})$ is a solution of (5), as required.

\section{A Priori Bounds}

Lemma 1. Let $\alpha \leq \beta$ be strict lower and strict upper solutions for (1) on $[0,1]$ and let $\boldsymbol{\alpha}$ and $\boldsymbol{\beta}$ be given by (14) and (15). There exists $\delta_{1}>0$ such that if $h=1 / n<\delta_{1}$, then $\boldsymbol{\alpha}$ and $\boldsymbol{\beta}$ are strict discrete lower and strict discrete upper solutions, respectively, for (9). 
Proof Now $\alpha_{k}=\alpha\left(t_{k}\right), \beta_{k}=\beta\left(t_{k}\right)$. By the Mean Value Theorem,

$$
\begin{array}{r}
\left|D \alpha_{k}\right|,\left|D \beta_{k}\right| \leq \max \left\{\left|\alpha^{\prime}(t)\right|,\left|\beta^{\prime}(t)\right|: t \in[0,1]\right\}+1=L, \\
\left|D^{2} \alpha_{k+1}\right|,\left|D^{2} \beta_{k+1}\right| \leq \max \left\{\left|\alpha^{\prime \prime}(t)\right|,\left|\beta^{\prime \prime}(t)\right|: t \in[0,1]\right\}+1=P .
\end{array}
$$

By Taylor's Theorem, $\alpha\left(t_{k-1}\right)=\alpha\left(t_{k}\right)-h \alpha^{\prime}\left(t_{k}\right)+O\left(h^{2}\right)$, thus $\left|D \alpha_{k}-\alpha^{\prime}\left(t_{k}\right)\right| \leq K h$ and hence $\left|D \alpha_{k}-\alpha^{\prime}\left(t_{k}\right)\right|$ converges uniformly to zero as $h$ goes to zero. By Taylor's Theorem,

$$
\begin{aligned}
& \alpha\left(t_{k+1}\right)=\alpha\left(t_{k}\right)+h \alpha^{\prime}\left(t_{k}\right)+\frac{h^{2}}{2} \alpha^{\prime \prime}\left(t_{k}\right)+o\left(h^{2}\right) \\
& \alpha\left(t_{k-1}\right)=\alpha\left(t_{k}\right)-h \alpha^{\prime}\left(t_{k}\right)+\frac{h^{2}}{2} \alpha^{\prime \prime}\left(t_{k}\right)+o\left(h^{2}\right) .
\end{aligned}
$$

By (28), (29) $\left|D^{2} \alpha_{k+1}-\alpha^{\prime \prime}\left(t_{k}\right)\right| \leq \eta(h)$ where we write $q=o(h)$ when $q=\eta(h) h$ and $\eta(h) \rightarrow 0$ as $h \rightarrow 0$. Thus $\left|D^{2} \alpha_{k+1}-\alpha^{\prime \prime}\left(t_{k}\right)\right|$ converges uniformly to zero as $h \rightarrow 0$.

Then by the uniform continuity of $f$ on bounded subset of $[0,1] \times \mathbb{R}^{2}$, there is $\delta_{1}$ such that $0<h<\delta_{1}$

$$
\begin{aligned}
& D^{2} \alpha_{k+1}-\left(\frac{f\left(t_{k+1}, \alpha_{k+1}, D \alpha_{k+1}\right)}{4}+\frac{2 f\left(t_{k}, \alpha_{k}, D \alpha_{k}\right)}{4}+\frac{f\left(t_{k-1}, \alpha_{k-1}, D \alpha_{k-1}\right)}{4}\right) \\
& \geq \alpha^{\prime \prime}\left(t_{k}\right)-\left(\frac{f\left(t_{k+1}, \alpha\left(t_{k+1}\right), \alpha^{\prime}\left(t_{k+1}\right)\right)}{4}+\frac{2 f\left(t_{k}, \alpha\left(t_{k}\right), \alpha^{\prime}\left(t_{k}\right)\right)}{4}\right. \\
& \left.\quad+\frac{f\left(t_{k-1}, \alpha\left(t_{k-1}\right), \alpha^{\prime}\left(t_{k-1}\right)\right)}{4}\right)-\frac{\gamma}{2} \\
& \geq \frac{\gamma}{2}
\end{aligned}
$$

In addition, we may choose $\delta_{1}$ such that for $0<h<\delta_{1}$

$$
\begin{aligned}
& \frac{f\left(t_{k+1}, \beta_{k+1}, D \beta_{k+1}\right)}{4}+\frac{2 f\left(t_{k}, \beta_{k}, D \beta_{k}\right)}{4}+\frac{f\left(t_{k-1}, \beta_{k-1}, D \beta_{k-1}\right)}{4}-D^{2} \beta_{k+1} \\
& \geq\left(\frac{f\left(t_{k+1}, \beta\left(t_{k+1}\right), \beta^{\prime}\left(t_{k+1}\right)\right)}{4}+\frac{2 f\left(t_{k}, \beta\left(t_{k}\right), \beta^{\prime}\left(t_{k}\right)\right.}{4}\right. \\
& \left.\quad+\frac{f\left(t_{k-1}, \beta\left(t_{k-1}\right), \beta^{\prime}\left(t_{k-1}\right)\right)}{4}\right)-\beta^{\prime \prime}\left(t_{k+1}\right)-\frac{\gamma}{2} \\
& \geq \frac{\gamma}{2} .
\end{aligned}
$$

The following Lemma concerns solutions to the modified difference equations.

Lemma 2. Let $\boldsymbol{\alpha} \leq \boldsymbol{\beta}$ be nondegenerate strict discrete lower and strict discrete upper solutions for (9). Assume that there is a constant $K>0$ such that $|\tilde{f}(t, y, p)|<K$ for all $(t, y, p) \in[0,1] \times \mathbb{R}^{2}$. Assume that $\tilde{f}\left(t_{j}, \alpha_{j}, p\right) \geq \tilde{f}\left(t_{j}, y_{j}, p\right)$ for all $j \in\{0, \cdots, n\}, \alpha_{j} \geq y_{j}$ and $p \in \mathbb{R}$ and $\tilde{f}\left(t_{j}, \beta_{j}, p\right) \leq \tilde{f}\left(t_{j}, y_{j}, p\right)$ for all $j \in\{0, \cdots, n\}, \beta_{j} \leq y_{j}$ and $p \in \mathbb{R}$. Then there exist $\delta_{2}>0$ such that for $0<h=1 / n<\delta_{2}$, solutions $(\mathbf{y}, \mathbf{z})$ of

$$
\begin{aligned}
& D y_{k}=\left(z_{k}+z_{k-1}\right) / 2, \\
& D z_{k}=\left(\tilde{f}_{k}+\tilde{f}_{k-1}\right) / 2,
\end{aligned}
$$

satisfying $\alpha_{0} \leq y_{0} \leq \beta_{0}$ and $\alpha_{n} \leq y_{n} \leq \beta_{n}$, satisfy $\boldsymbol{\alpha} \leq \mathbf{y} \leq \boldsymbol{\beta}$.

Proof Let $\mathbf{y}$ and $\mathbf{z}$ be solutions of (30), (31) satisfying $\left(y_{0}, y_{n}\right) \in \bar{\triangle}$. We show that $\boldsymbol{\alpha} \leq \mathbf{y} \leq \boldsymbol{\beta}$. Suppose that $\alpha_{t}>y_{t}$ for some $t \in\{0, \cdots, n\}$. Since $\alpha_{0} \leq y_{0} \leq \beta_{0}, \alpha_{n} \leq y_{n} \leq \beta_{n}$ then we may assume $\alpha_{t}-y_{t}$ has maximum at $k$ for some $0<k<n$ with $\alpha_{k}-y_{k}>0$. This implies $D\left(\alpha_{k}-y_{k}\right) \geq 0 \geq D\left(\alpha_{k+1}-y_{k+1}\right)$. Hence

$$
D^{2} \alpha_{k+1}-D^{2} y_{k+1} \leq 0
$$

Also we have

$$
\begin{aligned}
-D y_{k} & =-D y_{k+1}+h\left(\tilde{f}_{k+1}+2 \tilde{f}_{k}+\tilde{f}_{k-1}\right) / 4 \\
& \leq-D y_{k+1}+h K .
\end{aligned}
$$


Thus

$$
\begin{aligned}
0<D \alpha_{k}-D y_{k} & \leq D \alpha_{k}-D y_{k+1}+h K \\
& \leq D \alpha_{k}-D \alpha_{k+1}+h K \\
& \leq h\left(\max \left|\alpha^{\prime \prime}(t)\right|+K\right) \\
& \leq h(P+K)<\delta_{2}(P+K), \text { for } h<\delta_{2},
\end{aligned}
$$

and

$$
\begin{aligned}
\left|D \alpha_{j}-D y_{j}\right| & \leq\left|D \alpha_{k}-D y_{k}\right|+\left|D \alpha_{j}-D \alpha_{k}\right|+\left|D y_{k}-D y_{j}\right| \\
& \leq h(2 P+2 K)<\delta_{2}(2 P+2 K), \text { for } h<\delta_{2}
\end{aligned}
$$

if $j=k-1$ or $j=k+1$. By the uniform continuity of $\tilde{f}$ on bounded subsets of $[0,1] \times \mathbb{R}^{2}$, we may choose $\delta_{2} \in\left(0, \delta_{1}\right)$ such that for all points $(t, y, p)$ and $(t, u, v) \in[0,1] \times\left[\alpha_{m}, \beta_{M}\right] \times[-L, L]$ with $|y-u|<\delta_{2}$ and $|p-v|<\delta_{2}(2 P+2 K)$, we have $|\tilde{f}(t, y, p)-\tilde{f}(t, u, v)|<\gamma$; here $\delta_{1}, P$ and $L$ are given in Lemma 1 .

From the definition of lower solution $\alpha_{k}$, for $\delta_{2}$ sufficiently small thus we obtain

$$
\begin{aligned}
& D^{2} \alpha_{k+1}-D^{2} y_{k+1} \\
& \geq \frac{\tilde{f}\left(t_{k+1}, \alpha_{k+1}, D \alpha_{k+1}\right)+2 \tilde{f}\left(t_{k}, \alpha_{k}, D \alpha_{k}\right)+\tilde{f}\left(t_{k-1}, \alpha_{k-1}, D \alpha_{k-1}\right)}{4}+\gamma \\
& -\frac{\tilde{f}\left(t_{k+1}, y_{k+1}, D y_{k+1}\right)+2 \tilde{f}\left(t_{k}, y_{k}, D y_{k}\right)+\tilde{f}\left(t_{k-1}, y_{k-1}, D y_{k-1}\right)}{4} .
\end{aligned}
$$

Now $y_{k}<\alpha_{k}$, so $\tilde{f}\left(t_{k}, y_{k}, D y_{k}\right)>\tilde{f}\left(t_{k}, \alpha_{k}, D \alpha_{k}\right)-\gamma$ as $\left|D y_{k}-D \alpha_{k}\right|<\delta_{2}(2 P+2 K)$. Similarly if $y_{j} \leq \alpha_{j}$ for $j=k-1$ or $j=k+1$ then $\tilde{f}\left(t_{j}, y_{j}, D y_{j}\right)>\tilde{f}\left(t_{j}, \alpha_{j}, D \alpha_{j}\right)-\gamma$ as $\left|D y_{j}-D \alpha_{j}\right|<\delta_{2}(2 P+2 K)$. Moreover if $y_{j}>\alpha_{j}$ with $j=k-1$ or $j=k+1$ then $\left|y_{j}-\alpha_{j}\right|<\delta_{2}$ and $\left|D y_{j}-D \alpha_{j}\right|<\delta_{2}(2 P+2 K)$, thus $\tilde{f}\left(t_{j}, y_{j}, D y_{j}\right)>\tilde{f}\left(t_{j}, \alpha_{j}, D \alpha_{j}\right)-\gamma$.

$$
\begin{aligned}
& \therefore D^{2} \alpha_{k+1}-D^{2} y_{k+1} \\
& >\frac{\tilde{f}\left(t_{k+1}, \alpha_{k+1}, D \alpha_{k+1}\right)+2 \tilde{f}\left(t_{k}, \alpha_{k}, D \alpha_{k}\right)+\tilde{f}\left(t_{k-1}, \alpha_{k-1}, D \alpha_{k-1}\right)}{4}+\gamma \\
& -\frac{\tilde{f}\left(t_{k+1}, \alpha_{k+1}, D \alpha_{k+1}\right)+2 \tilde{f}\left(t_{k}, \alpha_{k}, D \alpha_{k}\right)+\tilde{f}\left(t_{k-1}, \alpha_{k-1}, D \alpha_{k-1}\right)}{4}-\gamma \\
& =0 .
\end{aligned}
$$

Hence $D^{2} \alpha_{k+1}-D^{2} y_{k+1}>0$ which is a contradiction to (32). Thus $\alpha_{j} \leq y_{j}$ for $j \in\{0, \cdots, n\}$. Similarly $\beta_{j} \geq y_{j}$. Thus $\boldsymbol{\alpha} \leq \mathbf{y} \leq \boldsymbol{\beta}$.

The following Nagumo style result is an analogue of ( Thompson, 1996, Theorem 2.1)

Theorem 3. Let $\boldsymbol{\alpha} \leq \boldsymbol{\beta}$ be strict discrete lower and strict discrete upper solutions for (9). Let $f_{k}$ satisfy the discrete Bernstein-Nagumo condition for $k=1, \cdots, n$. If $\mathbf{y}$ is a solution of (5)-(7) with $\boldsymbol{\alpha} \leq \mathbf{y} \leq \boldsymbol{\beta}$ then $\left|z_{k}\right| \leq N$ for $k=1, \cdots, n$ where $N$ is given in (19).

Proof Let $\beta_{M}=\max \left\{\beta_{k}: k=0, \cdots, n\right\}, \alpha_{m}=\min \left\{\alpha_{k}: k=0, \cdots, n\right\}$. Choose $\epsilon>0$ so that

$$
\int_{\sigma}^{N} \frac{s d s}{h(s)}>\beta_{M}-\alpha_{m}+\epsilon .
$$

Suppose that $\left|z_{k}\right| \leq N$ for all $\mathrm{k}$ is false. Thus there is $k_{0}$ such that $\left|z_{k_{0}}\right|>N$. We consider the case $z_{k_{0}}>N$; the case $z_{k_{0}}<-N$ is similar. We show there is $k_{1}$ such that $0 \leq z_{k_{1}} \leq \sigma$. First consider the subcase $z_{k} \geq 0$ for all $k, 0 \leq k \leq n$. Then

$$
\sigma \geq\left|y_{n}-y_{0}\right|=\left|\sum_{i=1}^{n} h D y_{i}\right|=\sum_{i=1}^{n} \frac{h}{2}\left(z_{i}+z_{i-1}\right) .
$$

Thus $z_{k_{1}} \leq \sigma$ for some $k_{1}$. 
Now consider the subcase $z_{k_{2}}<0$ for some $k_{2}$. Assume $k_{2}<k_{0}$; the subcase $k_{2}>k_{0}$ is similar. Thus there is $k_{1}>k_{2}$ such that $z_{k_{1}-1}<0 \leq z_{k_{1}}$. But by (6) and the continuity of $f$ over the bounded interval $[0,1]$, say $|f| \leq K$ implies $\left|p_{k}\right| \leq K$. Thus $\left|z_{k}-z_{k-1}\right|=h\left|p_{k}\right| \leq h K$ for $h \leq \frac{\sigma}{K}$, and thus

$$
0 \leq z_{k_{1}}=z_{k_{1}-1}+\left(z_{k_{1}}-z_{k_{1}-1}\right) \leq z_{k_{1}}-z_{k_{1}-1} \leq h K \leq \sigma
$$

as required. Proceeding with this argument we may choose $k_{1}$ and $k_{3}$ such that $0 \leq z_{k_{1}} \leq \sigma<N \leq z_{k_{3}}$ and $\sigma \leq z_{k} \leq N$ for $k_{1}<k<k_{3}$. Let $W=\left\{i: k_{1}<i \leq k_{3}, z_{i}>z_{i-1}\right\}$. Thus $[\sigma, N] \subseteq \cup_{i \in \bar{W}}\left[z_{i-1}, z_{i}\right]$. Let $\bar{W} \subseteq W$ be a minimal subset of $W$ such that $[\sigma, N] \subseteq \cup_{i \in \bar{W}}\left[z_{i-1}, z_{i}\right]$. Hence $\left[\sigma^{2}, N^{2}\right] \subseteq \cup_{i \in \bar{W}}\left[z_{i-1}^{2}, z_{i}^{2}\right]$.

Now

$$
\begin{aligned}
\int_{\sigma}^{N} \frac{s d s}{\phi(s)} & =\int_{\sigma^{2}}^{N^{2}} \frac{d \tau}{2 \phi(\sqrt{ } \tau)} \\
& \leq \sum_{i \in \bar{W}} \int_{z_{i-1}^{2}}^{z_{i}^{2}} \frac{d \tau}{2 \phi(\sqrt{ } \tau)} \\
& \leq \sum_{i \in \bar{W}} \frac{z_{i}^{2}-z_{i-1}^{2}}{2 \phi\left(\sqrt{ } z_{i-1}^{2}\right)} \\
& =\sum_{i \in \bar{W}} \frac{z_{i}^{2}-z_{i-1}^{2}}{2 \phi\left(z_{i-1}\right)}
\end{aligned}
$$

Here we use $\phi(\sqrt{ } \tau)$ is non-decreasing and $0 \leq z_{i-1}^{2} \leq z_{i}^{2}$

$$
\leq \sum_{i \in \bar{W}} \frac{z_{i}^{2}-z_{i-1}^{2}}{2 \phi\left(\sqrt{ } z_{i}^{2}\right)}+\sum_{i \in \bar{W}} \frac{z_{i}^{2}-z_{i-1}^{2}}{2}\left\{\frac{1}{\phi\left(z_{i-1}\right)}-\frac{1}{\phi\left(z_{i}\right)}\right\} \text {. }
$$

Now

$$
\sum_{i \in \bar{W}} \frac{z_{i}^{2}-z_{i-1}^{2}}{2 \phi\left(z_{i}\right)}=\sum_{i \in \bar{W}} \frac{z_{i}+z_{i-1}}{2} h \frac{D z_{i}}{\phi\left(z_{i}\right)} \leq \sum_{i \in \bar{W}} h \frac{z_{i}+z_{i-1}}{2}
$$

as

$$
\left|D z_{i}\right| \leq \frac{1}{2}\left(\left|f_{i}\right|+\left|f_{i-1}\right|\right) \leq \frac{1}{2}\left(\phi\left(z_{i}\right)+\phi\left(z_{i-1}\right)\right) \leq \phi\left(z_{i}\right)
$$

as $0 \leq z_{i-1} \leq z_{i}$, and $\phi$ is a non-decreasing function. Moreover since $\bar{W}$ is minimal, $\bar{W}=\bar{W}_{a} \cup \bar{W}_{b}$ where $\left(z_{i-1}, z_{i}\right) \cap$ $\left(z_{j-1}, z_{j}\right) \neq \varnothing, \forall i \neq j, i, j \in \bar{W}_{a}$ and similarly for $i \neq j, i, j \in \bar{W}_{b}$. Now $\phi(\sigma) \leq \phi\left(z_{i-1}\right), \forall i \in \bar{W}$. Thus

$$
\begin{aligned}
& \sum_{i \in \bar{W}_{a}} \frac{z_{i}^{2}-z_{i-1}^{2}}{2}\left\{\frac{1}{\phi\left(z_{i-1}\right)}-\frac{1}{\phi\left(z_{i}\right)}\right\} \\
& \leq \sum_{i \in \bar{W}_{a}} \frac{\left(z_{i}+z_{i-1}\right)\left(z_{i}-z_{i-1}\right)}{2}\left\{\frac{1}{\phi\left(z_{i-1}\right)}-\frac{1}{\phi\left(z_{i}\right)}\right\} \\
& \leq(N+1) \max \left\{\left|z_{i}-z_{i-1}\right|\right\} \frac{1}{\phi(\sigma)} \\
& \leq \frac{(N+1) K h}{\phi(\sigma)}
\end{aligned}
$$

since $z_{k} \leq N+1$ for all $k$ when $h K \leq 1$, so

$$
\left|\left(z_{i}+z_{i-1}\right) / 2\right| \leq N+1 \text { and }\left|z_{i}-z_{i-1}\right|=h\left|p_{i}\right| \leq h K
$$

Similarly

$$
\sum_{i \in \bar{W}_{b}} \frac{z_{i}^{2}-z_{i-1}^{2}}{2}\left\{\frac{1}{\phi\left(z_{i-1}\right)}-\frac{1}{\phi\left(z_{i}\right)}\right\} \leq \frac{(N+1) K h}{\phi(\sigma)}
$$


Thus

$$
\begin{aligned}
& \int_{\sigma}^{N} \frac{s d s}{\phi(s)} \\
& \leq \sum_{i \in \bar{W}} h \frac{\left(z_{i}+z_{i-1}\right)}{2}+\frac{2(N+1) K h}{\phi(\sigma)} \\
& \leq \sum_{i \in \bar{W}}\left(y_{i}-y_{i-1}\right)+\frac{2(N+1) K h}{\phi(\sigma)} \\
& \leq \sum_{i=k_{0+1}}^{k_{2}}\left(y_{i}-y_{i-1}\right)+\epsilon
\end{aligned}
$$

when $\frac{2(N+1) h K}{\phi(\sigma)}<\epsilon$. Since $0 \leq\left(z_{i}+z_{i-1}\right) / 2$ for $k_{1}+1 \leq i \leq k_{3}$, thus $y_{i} \geq y_{i-1}$ for $k_{1}+1 \leq i \leq k_{3}$. Hence

$$
\int_{\sigma}^{N} \frac{s d s}{\phi(s)} \leq y_{k_{2}}-y_{k_{0}}+\epsilon \leq \beta_{M}-\alpha_{m}+\epsilon
$$

which is a contradiction to (33), and the result follows.

\section{Nonlinear Boundary Conditions and Compatibility}

Let $\boldsymbol{\alpha} \leq \boldsymbol{\beta}$ be non-degenerate, strict discrete lower and strict discrete upper solutions, respectively for (9).

Definition 4. (Henderson and Thompson, 2001) We call the vector field $\psi=\left(\psi^{0}, \psi^{1}\right) \in C\left(\bar{\triangle} ; \mathbb{R}^{2}\right)$ discrete inwardly pointing on $\triangle$ if for all $(C, D) \in \partial \triangle$

$$
\psi^{0}\left(\alpha_{0}, D\right) \geq D \alpha_{k}, \quad \psi^{0}\left(\beta_{0}, D\right) \leq D \beta_{k}
$$

and

$$
\psi^{1}\left(C, \alpha_{n}\right) \leq D \alpha_{n}, \quad \psi^{1}\left(C, \beta_{n}\right) \geq D \beta_{n}
$$

We call $\psi$ strongly discrete inwardly pointing if the weak inequalities are replaced by strict inequalities.

Definition 5. (Henderson and Thompson, 2001) Let $G \in C\left(\bar{\Delta} \times \mathbb{R}^{2} ; \mathbb{R}^{2}\right)$. We say $G$ is very strongly (strongly) discrete compatible at level $L$ with $\boldsymbol{\alpha}$ and $\boldsymbol{\beta}$ if for all discrete (strongly discrete) inwardly pointing $\psi$ on $\Delta$ satisfying $|\psi| \leq L$,

$$
\mathcal{G}(C, D) \neq 0 \text { for all }(C, D) \in \partial \triangle
$$

and

$$
d(G, \triangle, 0) \neq 0
$$

where

$$
\mathcal{G}(C, D)=G((C, D) ; \psi(C, D)) \text { for all }(C, D) \in \bar{\triangle}
$$

If it is very strongly (strongly) discrete compatible at all levels then we simply say it is very strongly (strongly) discrete compatible with $\boldsymbol{\alpha}$ and $\boldsymbol{\beta}$.

Remark 4. If $G$ is very strongly (strongly) discrete compatible with $\boldsymbol{\alpha}$ and $\boldsymbol{\beta}$ then the Brouwer degree (38) is independent of the (strongly) inwardly pointing vector field $\psi$. It is not difficult to see that (strongly) inwardly pointing vector fields always exist. Moreover, since we consider very strongly discrete compatible boundary conditions $G$, it follows that (38) holds for all discrete inwardly pointing vector fields, and so we will choose $\psi$ as follows:

$$
\psi^{0}(C, D)=\left(\beta_{1}-\beta_{0}\right) / h \frac{C-\alpha_{0}}{\beta_{0}-\alpha_{0}}+\left(\alpha_{1}-\alpha_{0}\right) / h \frac{\beta_{0}-C}{\beta_{0}-\alpha_{0}}
$$

and

$$
\psi^{1}(C, D)=\left(\beta_{n}-\beta_{n-1}\right) / h \frac{D-\alpha_{n}}{\beta_{n}-\alpha_{n}}+\left(\alpha_{n}-\alpha_{n-1}\right) / h \frac{\beta_{n}-D}{\beta_{n}-\alpha_{n}}
$$


For the linear Sturm-Liouville boundary conditions, $G=\left(g^{0}, g^{1}\right)$ where

$$
g^{0}\left(\left(y_{0}, y_{n}\right),\left(\left(z_{0}+z_{1}\right) / 2,\left(z_{n-1}+z_{n}\right) / 2\right)\right)=a_{0} y_{0}-a_{1}\left(z_{0}+z_{1}\right) / 2-A=0
$$

and

$$
g^{1}\left(\left(y_{0}, y_{n}\right),\left(\left(z_{0}+z_{1}\right) / 2,\left(z_{n-1}+z_{n}\right) / 2\right)\right)=b_{0} y_{n}+b_{1}\left(z_{n-1}+z_{n}\right) / 2-B=0
$$

where $a_{0}+b_{0}>0, a_{0}+a_{1}>0$, and $b_{0}+b_{1}>0$. These include the Picard (also called Dirichlet) boundary conditions in the special case $a_{0}=1=b_{0}$ and $a_{1}=0=b_{1}$. For the Neumann boundary conditions

$$
g^{0}\left(\left(y_{0}, y_{n}\right),\left(\left(z_{0}+z_{1}\right) / 2,\left(z_{n-1}+z_{n}\right) / 2\right)\right)=\left(z_{0}+z_{1}\right) / 2-A=0
$$

and

$$
g^{1}\left(\left(y_{0}, y_{n}\right),\left(\left(z_{0}+z_{1}\right) / 2,\left(z_{n-1}+z_{n}\right) / 2\right)\right)=\left(z_{n-1}+z_{n}\right) / 2-B=0
$$

while for the periodic boundary conditions

$$
g^{0}\left(\left(y_{0}, y_{n}\right),\left(\left(z_{0}+z_{1}\right) / 2,\left(z_{n-1}+z_{n}\right) / 2\right)\right)=y_{0}-y_{n}=0
$$

and

$$
g^{1}\left(\left(y_{0}, y_{n}\right),\left(\left(z_{0}+z_{1}\right) / 2,\left(z_{n-1}+z_{n}\right) / 2\right)\right)=\left(z_{n-1}+z_{n}\right) / 2-\left(z_{0}+z_{1}\right) / 2=0 .
$$

For these boundary conditions it is not difficult to show that the very strong discrete compatibility conditions become the familiar ones usually assumed in the presence of lower and upper solutions; that is,

$$
\begin{aligned}
& a_{0} \alpha_{1}-a_{1} D \alpha_{1}<A<a_{0} \beta_{1}-a_{1} D \beta_{1}, \\
& b_{0} \alpha_{n}+b_{1} D \alpha_{n}<B<b_{0} \beta_{n}+b_{1} D \beta_{n}
\end{aligned}
$$

for the linear Sturm-Liouville boundary conditions,

$$
D \alpha_{1}>A, D \beta_{1}<A, D \alpha_{n}<B, D \beta_{n}>B
$$

for the Neumann boundary conditions, and

$$
\alpha_{1}=\alpha_{n}, \beta_{1}=\beta_{n}, D \alpha_{1}>D \alpha_{n}, D \beta_{1}<D \beta_{n}
$$

for the periodic boundary conditions.

We will need the following result of (Thompson, 1996).

Lemma 5. Let $\alpha \leq \beta$ be strict lower and strict upper solutions for (1) on $[0,1]$, and let $G \in C\left(\bar{\Delta} \times \mathbb{R}^{2} ; \mathbb{R}^{2}\right)$ be very strongly compatible with $\alpha$ and $\beta$. Let $\boldsymbol{\alpha}$ and $\boldsymbol{\beta}$ be the strict discrete lower and strict discrete upper solutions for (9) given in Lemma 1 and $N>0$. There exists $\delta_{3} \in\left(0, \delta_{1}\right)$ such that if $0<h=\frac{1}{n}<\delta_{3}$, and $\Psi=\left(\psi^{0}, \psi^{1}\right) \in C\left(\bar{\Delta} ; \mathbb{R}^{2}\right)$ satisfies

$$
\begin{aligned}
& |\Psi(C, D)| \leq N, \\
& \psi^{0}\left(\alpha_{0}, D\right) \geq D \alpha_{1}, \psi^{0}\left(\beta_{0}, D\right) \leq D \beta_{1} \text { and } \\
& \psi^{1}\left(C, \alpha_{n}\right) \leq D \alpha_{n}, \psi^{1}\left(C, \beta_{n}\right) \geq D \beta_{n},
\end{aligned}
$$

on $\bar{\Delta}$, then

$$
\begin{aligned}
\mathcal{G}(C, D) & \neq 0 \text { for all }(C, D) \in \partial \Delta \text { and } \\
d(\mathcal{G}, \Delta, 0) & \neq 0
\end{aligned}
$$

where

$$
\mathcal{G}(C, D)=G((C, D) ; \Psi(C, D)) \text { for all }(C, D) \in \bar{\Delta}
$$

We call $G$ satisfying (45) for $\Psi$ satisfying (41) through (43) very strongly discrete compatible with $\boldsymbol{\alpha}$ and $\boldsymbol{\beta}$ at level $N$.

\section{Existence of Solutions}

We now present our main result. 
Theorem 6. Assume that there exist non-degenerate strict lower and strict upper solutions $\alpha \leq \beta$ for (9), that the $f$ satisfies the Bernstein-Nagumo condition and that $G \in C\left(\bar{\Delta} \times \mathbb{R}^{2}, \mathbb{R}^{2}\right)$ is very strongly compatible with $\alpha$ and $\beta$. Then there exists $\delta>0$ such that for $0<h=1 / n<\delta$ there exist solutions $\mathbf{y}$ and $\mathbf{z}$ of problems (5), (6) and (7) with $\boldsymbol{\alpha} \leq \mathbf{y} \leq \boldsymbol{\beta}$ and $\left|z_{k}\right|<L+1$ for $k=1, \cdots, n$ and $\left|z_{0}\right|<2 \frac{\beta_{M}-\alpha_{m}+2 \epsilon}{h}+L+1$.

\section{Proof Choose}

$$
L>\max \left\{\left|\alpha^{\prime}(t)\right|,\left|\beta^{\prime}(t)\right|, \sigma,|\psi(C, D)|:(C, D) \in \bar{\triangle}, t \in[0,1]\right\}+1,
$$

and $\epsilon>0$ such that

$$
\int_{\sigma}^{L-1} \frac{s d s}{\phi(s)+\epsilon}>\beta_{M}-\alpha_{m}+2 \epsilon=\beta_{\epsilon}-\alpha_{\epsilon}
$$

where $\alpha_{m}=\min \{\alpha(t): t \in[0,1]\}, \beta_{M}=\max \{\beta(t): t \in[0,1]\}, \alpha_{\epsilon}:=\alpha_{m}-\epsilon, \beta_{\epsilon}:=\beta_{M}+\epsilon, \boldsymbol{\alpha}_{\epsilon, j}:=\alpha_{\epsilon}$ and $\boldsymbol{\beta}_{\epsilon, j}:=\beta_{\epsilon}$, for $j \in\{0, \cdots, n\}$.

By Lemmas 1 and 5 we may choose $\delta>0$ sufficiently small that for $0<h=1 / n<\delta, \boldsymbol{\alpha}$ and $\boldsymbol{\beta}$ given by (14) and (15) are strict discrete lower and strict discrete upper solutions for (5) that are very strongly discrete compatible with $G$ at level $L$. Let $\Psi=\left(\psi^{0}, \psi^{1}\right)$ be the discrete inwardly pointing vector field on $\bar{\Delta}$ with $|\Psi| \leq L$. We modify $f_{k}$ for $\mathbf{y}$ not between $\boldsymbol{\alpha}$ and $\boldsymbol{\beta}$. We show that a solution of the modified problem lies in the region where $f_{k}, k=0, \cdots, n$ is unmodified and hence is the required solution. We use Brouwer degree theory to prove existence for the modified problem and compute the degree using a homotopy; the modification is chosen to facilitate the construction of a suitable homotopy.

Consider the BVP

$$
\begin{aligned}
D^{2} y_{k+1} & =\left(1-\left|T\left(y_{k}, \alpha_{k}, \beta_{k}, \epsilon\right)\right|\right)\left(\tilde{f}_{k+1}+2 \tilde{f}_{k}+\tilde{f}_{k-1}\right) / 4+ \\
T & \left(y_{k}, \alpha_{k}, \beta_{k}, \epsilon\right)\left(\left|\left(\tilde{f}_{k+1}+2 \tilde{f}_{k}+\tilde{f}_{k-1}\right) / 4\right|+\epsilon\right)=: \tilde{F}_{k}, 1 \leq k \leq n-1 \\
z_{k} & =\sum_{i=1}^{k}\left(\tilde{f}_{i}+\tilde{f}_{i-1}\right) / 2+z_{0}, \quad 1 \leq k \leq n \\
z_{0} & =2\left(y_{1}-y_{0}\right) / h-z_{1}
\end{aligned}
$$

together with boundary conditions (7) where $\pi, T, p_{i}$ and $\tilde{f}_{k}$ are given in (20), (21), (6) and (22) respectively.

Clearly $\tilde{F}_{k}$ is a bounded continuous function on $\mathbb{R}^{n+1} \times \mathbb{R}^{n+1}$, and satisfies Nagumo condition in $\Omega_{\epsilon}$. In particular

$$
\begin{aligned}
\left|D^{2} y_{k+1}\right| & \leq\left|\left(\tilde{f}_{k+1}+2 \tilde{f}_{k}+\tilde{f}_{k-1}\right) / 4\right|+\epsilon \text { so that } \\
& \leq \frac{1}{4}\left(\phi\left(\left|z_{k+1}\right|\right)+2 \phi\left(\left|z_{k}\right|\right)+\phi\left(\left|z_{k-1}\right|\right)\right)+\epsilon
\end{aligned}
$$

and

$$
\left|D^{2} y_{k+1}\right| \leq \phi(L)+\epsilon=K, \text { for all }(y, z)
$$

Now $\boldsymbol{\alpha}_{\epsilon}=\left(\alpha_{\epsilon, 0}, \cdots, \alpha_{\epsilon, n}\right)$ is a strict discrete lower solution for the problem (48), (49). Since

$$
D^{2} \boldsymbol{\alpha}_{\epsilon, k+1}=0>-\left(\left|\left(\left(\tilde{f} \boldsymbol{\alpha}_{\epsilon}\right)_{k+1}+2\left(\tilde{f} \boldsymbol{\alpha}_{\epsilon}\right)_{k}+\left(\tilde{f} \boldsymbol{\alpha}_{\epsilon}\right)_{k-1}\right) / 4\right|+\epsilon\right)
$$

where $(f \mathbf{z})_{k}=f\left(t_{k}, z_{k}, D z_{k}\right)$, and

$$
\left(\tilde{f} \boldsymbol{\alpha}_{\epsilon}\right)_{k}=f\left(t_{k}, \pi\left(y_{k}, \alpha_{\epsilon}, \beta_{\epsilon}\right), \pi\left(D \boldsymbol{\alpha}_{\epsilon, k},-L, L\right)\right) .
$$

Since $\boldsymbol{\alpha}_{\epsilon}$ is a contant vector, $D \boldsymbol{\alpha}_{\epsilon}=0$ so $\boldsymbol{\alpha}_{\epsilon}$ is a strict discrete lower solution. Similarly $\boldsymbol{\beta}_{\epsilon}=\left(\beta_{\epsilon, 0}, \beta_{\epsilon, 1}, \cdots, \beta_{\epsilon, n}\right)$ is a strict discrete upper solution for this equation. Since $\left|D^{2} y_{k+1}\right| \leq K=\phi(L)+\epsilon$ for all $\left(t_{i}, y_{i}, z_{i}\right) \in[0,1] \times \mathbb{R}^{n+1} \times \mathbb{R}^{n+1}$ for all $\left|z_{i}\right| \leq L$, thus by Lemma 1, 2 and Theorem 3, the solutions $(\mathbf{y}, \mathbf{z})$ of (5) and (6) satisfy $\boldsymbol{\alpha} \leq \mathbf{y} \leq \boldsymbol{\beta}$ and $\left|z_{k}\right|<L+1$ for $k=1, \cdots, n$ and $\left|z_{0}\right|<2 \frac{\beta_{M}-\alpha_{m}+2 \epsilon}{h}+L+1$ when $\left(y_{0}, y_{n}\right) \in \bar{\triangle}$. As $\left(\tilde{f}_{k+1}+2 \tilde{f}_{k}+\tilde{f}_{k-1}\right) / 4$ and $\sum_{i=1}^{k}\left(\tilde{f}_{i}+\tilde{f}_{i-1}\right) / 2+z_{0}$ coincide with $\left(f_{k+1}+2 f_{k}+f_{k-1}\right) / 4$ and $\sum_{i=1}^{k}\left(f_{i}+f_{i-1}\right) / 2+z_{0}$ for $k=1, \cdots, n-1, i=1, \cdots, n$ in this region it suffices to show that there are solutions $(\mathbf{y}, \mathbf{z})$ of (48), (49), (50) and (7) with $\left(y_{0}, y_{n}\right) \in \bar{\triangle}$. Let

$$
\Omega_{\epsilon}=\left\{(\mathbf{y}, \mathbf{z}) \in \mathbb{R}^{n+1} \times \mathbb{R}^{n+1}: \alpha_{\epsilon, j}<y_{j}<\beta_{\epsilon, j},\left|z_{k}\right|<M \text { for } 0 \leq j \leq n, 0 \leq k \leq n\right\}
$$


where $M=2 \frac{\beta_{M}-\alpha_{m}+2 \epsilon}{h}+L+1$, and $\Gamma_{\epsilon}=\Omega_{\epsilon} \times \triangle$. Define $\mathcal{K}: \mathbb{R}^{n+1} \times \mathbb{R}^{n+1} \rightarrow \mathbb{R}^{n-1}, \mathcal{L}: \mathbb{R}^{n+1} \times \mathbb{R}^{n+1} \rightarrow \mathbb{R}^{n+1}$ by

$$
\begin{aligned}
& \mathcal{K}_{k}(\mathbf{y}, \mathbf{z})=\frac{\tilde{f}_{k+1}+2 \tilde{f}_{k}+\tilde{f}_{k-1}}{4} \\
& \left.\mathcal{L}_{i}(\mathbf{y}, \mathbf{z})\right)=\sum_{s=1}^{i} \frac{\tilde{f}_{s}+\tilde{f}_{s-1}}{2}+z_{0}
\end{aligned}
$$

for $k=1, \cdots, n-1, i=1, \cdots, n$ and $\mathcal{L}_{0}(\mathbf{y}, \mathbf{z})=0,(\mathbf{y}, \mathbf{z}) \in \mathbb{R}^{n+1} \times \mathbb{R}^{n+1}$. Define $\mathcal{H}: \bar{\Gamma}_{\epsilon} \times[0,1] \rightarrow \mathbf{X}$ by

$$
\begin{aligned}
& \mathcal{H}(\mathbf{y}, \mathbf{z}, C, D, \lambda) \\
& \left.=\left(\begin{array}{l}
\mathbf{y} \\
\mathbf{z}
\end{array}\right)-3 \lambda\left(\begin{array}{c}
\mathbf{w}(C, D) \\
\left(2\left(y_{1}-y_{0}\right) / h-z_{1}, 0, \cdots, 0\right)
\end{array}\right)-(1-3 \lambda)\left(\begin{array}{c}
\left(\bar{\alpha}_{\epsilon}+\bar{\beta}_{\epsilon}\right) / 2 \\
0
\end{array}\right), \mathcal{G}(C, D)\right) \\
& \text { for } 0 \leq \lambda \leq \frac{1}{3}, \\
& \mathcal{H}(\mathbf{y}, \mathbf{z}, C, D, \lambda) \\
& =\left(\left(\begin{array}{l}
\mathbf{y} \\
\mathbf{z}
\end{array}\right)-3(\lambda-1 / 3)\left(\begin{array}{c}
\mathbf{T}(\mathcal{K}(\mathbf{y}, \mathbf{z})) \\
\mathcal{L}(\mathbf{y}, \mathbf{z})
\end{array}\right)-\left(\begin{array}{c}
\left(2\left(y_{1}-y_{0}\right) / h-z_{1}, 0, \cdots, 0\right)
\end{array}\right), \mathcal{G}(C, D)\right) \\
& \text { for } \frac{1}{3} \leq \lambda \leq \frac{2}{3}, \text { and } \quad \mathbf{w}(C, D) \\
& \quad \mathcal{H}(\mathbf{y}, \mathbf{z}, C, D, \lambda) \\
& \quad=\left(\left(\begin{array}{l}
\mathbf{y} \\
\mathbf{z}
\end{array}\right)-\left(\begin{array}{c}
\mathbf{T}(\mathcal{K}(\mathbf{y}, \mathbf{z})) \\
\mathcal{L}(\mathbf{y}, \mathbf{z})
\end{array}\right)-\left(\begin{array}{c}
\left(2\left(y_{1}-y_{0}\right) / h-z_{1}, 0, \cdots, 0\right)
\end{array}\right), S(\mathbf{y}, \mathbf{z}, C, D, \lambda)\right) \\
& \quad \text { for } \frac{2}{3} \leq \lambda \leq 1,
\end{aligned}
$$

where $\psi$ is given by (39) and (40), $\boldsymbol{\alpha}_{\epsilon}=\left(\alpha_{\epsilon, 0}, \alpha_{\epsilon, 1}, \cdots, \alpha_{\epsilon, n}\right), \boldsymbol{\beta}_{\epsilon}=\left(\beta_{\epsilon, 0}, \beta_{\epsilon, 1}, \cdots, \beta_{\epsilon, n}\right)$, and

$$
S(\mathbf{y}, \mathbf{z}, C, D, \lambda)=G\left((C, D),\left(3(\lambda-2 / 3)\left(\left(z_{0}+z_{1}\right) / 2,\left(z_{n-1}+z_{n}\right) / 2\right)\right)+3(1-\lambda) \psi(C, D)\right)
$$

Clearly $\mathcal{H}$ is continuous. It is easy to see that $(\mathbf{y}, \mathbf{z})$ is a solution of (48), (49), (50) and (7) with $(\mathbf{y}, \mathbf{z}, C, D) \in \bar{\Gamma}_{\epsilon}$ iff $\mathcal{H}(\mathbf{y}, \mathbf{z}, C, D, 1)=0$. If there is a solution of $\mathcal{H}(\mathbf{y}, \mathbf{z}, C, D, 1)=0$ with $(\mathbf{y}, \mathbf{z}, C, D) \in \partial \Gamma_{\epsilon}$ then we are done. So we assume there is no such solution in $\partial \Gamma_{\epsilon}$. We show $\mathcal{H}$ is a homotopy for the Brouwer degree on $\Gamma_{\epsilon}$ at 0 . We argue by contradiction and assume solutions exist to $\mathcal{H}(\mathbf{y}, \mathbf{z}, C, D, \lambda)=0$ with $\lambda \in[0,1]$ and $(\mathbf{y}, \mathbf{z}, C, D) \in \partial \Gamma_{\epsilon}$.

We consider the cases $\lambda \in[2 / 3,1]$ and $\lambda \in[1 / 3,2 / 3)$; the case $\lambda \in[0,1 / 3)$ is trivial because $\mathcal{G}(C, D) \neq(0,0)$ with $(C, D) \in \partial \Delta$ and

$$
\left(\begin{array}{l}
\mathbf{y} \\
\mathbf{z}
\end{array}\right)-3 \lambda\left(\begin{array}{c}
\mathbf{w}(C, D) \\
2\left(y_{1}-y_{0}\right) / h-z_{1}, 0, \cdots, 0
\end{array}\right)-(1-3 \lambda)\left(\begin{array}{c}
\left(\bar{\alpha}_{\epsilon}+\bar{\beta}_{\epsilon}\right) / 2 \\
0
\end{array}\right) \neq\left(\begin{array}{l}
0 \\
0
\end{array}\right)
$$

for $(\mathbf{y}, \mathbf{z}) \in \partial \Omega_{\epsilon}$.

Case (i) $\lambda \in[2 / 3,1]$.

By assumption there is no solution with $\lambda=1$. Assume there is solution $(\mathbf{y}, \mathbf{z}, C, D)$ with $\lambda \in[2 / 3,1)$. We assume as before that $\boldsymbol{\alpha} \leq \mathbf{y} \leq \boldsymbol{\beta}, y_{0}=C$ and $y_{n}=D$. Assume that $\left(y_{0}, y_{n}\right) \in \partial \triangle$. If $y_{0}=\alpha_{0}$, then $\left(z_{0}+z_{1}\right) / 2 \geq D \alpha_{1}$. Thus

$$
3(\lambda-2 / 3)\left(z_{0}+z_{1}\right) / 2+3(1-\lambda) \varphi^{0}\left(y_{0}, y_{n}\right) \geq D \alpha_{1}
$$

since $\Psi$ is strongly inwardly pointing. It follows from the very strong discrete compatibility of $G$ that $\mathcal{S}(\mathbf{y}, \mathbf{z}, C, D, \lambda) \neq$ $(0,0)$, which is a contradiction. Similarly the other cases $(C, D)=\left(y_{0}, y_{n}\right) \in \partial \triangle$ also lead to a contradiction. Thus $(C, D) \notin \partial \triangle$.

Assume $\mathbf{y} \in \partial\left(\boldsymbol{\alpha}_{\epsilon}, \boldsymbol{\beta}_{\epsilon}\right)$, so either $y_{j}=\boldsymbol{\alpha}_{\epsilon, j}$ or $y_{j}=\boldsymbol{\beta}_{\epsilon, j}$ for some $j \in\{0, \cdots, n\}$. Assume $y_{j}=\boldsymbol{\alpha}_{\epsilon, j}$ for some $j \in\{0, \cdots, n\}$. Again from the proof of Lemma 2, we get a contradiction. Similarly $y_{j} \neq \boldsymbol{\beta}_{\epsilon, j}$ for some $j \in\{0, \cdots, n\}$. Hence $\mathbf{y} \notin \partial \Omega_{\epsilon}$.

Assume $\left|z_{j}\right|=2 \frac{\beta_{M}-\alpha_{m}+2 \epsilon}{h}+L+1$ for some $j, 0 \leq j \leq n$. By Theorem $3,\left|z_{j}\right| \leq \sigma<L+1$ for some $j \in\{1, \cdots, n\}$ and $\left|z_{0}\right|<2 \frac{\beta_{M}-\alpha_{m}+2 \epsilon}{h}+L+1$ which is a contradiction, where $z_{0}$ is given in (25). Thus $\mathbf{z} \notin \partial \Omega_{\epsilon}$. Thus there are no solutions of $\mathcal{H}(\mathbf{y}, \mathbf{z}, C, D)=0$ with $\lambda \in[2 / 3,1]$ and $(\mathbf{y}, \mathbf{z}, C, D) \in \partial \Gamma_{\epsilon}$.

Case (ii) $\lambda \in[1 / 3,2 / 3]$.

Since $G$ is very strongly discrete compatible, there is no solution $(\mathbf{y}, \mathbf{z}, C, D, \lambda)$ to $\mathcal{H}=0$ with $(C, D) \in \partial \Delta$. The proof of the case $(\mathbf{y}, \mathbf{z}) \in \partial \Omega_{\epsilon}$ leads to a contradiction in a similar way as for $[2 / 3,1)$. 
Thus $\mathcal{H}$ is a homotopy for the Brouwer degree and since $\mathcal{H}(., 0)=(I, \mathcal{G})$ where $I$ is the identity on $\mathbb{R}^{n+1} \times \mathbb{R}^{n+1}$ it follows that

$$
\begin{aligned}
d\left(\mathcal{H}(., 1), \Gamma_{\epsilon}, 0\right) & =d\left(\mathcal{H}(., 0), \Gamma_{\epsilon}, 0\right) \\
& =d(\mathcal{G}, \Delta,(0,0)) \neq 0 .
\end{aligned}
$$

Thus there is a solution $(\mathbf{y}, \mathbf{z}, C, D) \in \Gamma_{\epsilon}$ of $\mathcal{H}(\mathbf{y}, \mathbf{z}, C, D, 1)=0$ and hence a solution $(\mathbf{y}, \mathbf{z}) \in \mathbb{R}^{n+1} \times \mathbb{R}^{n+1}$ to the problem (5), (6) and (7).

Remark 7. Since $\boldsymbol{\alpha} \leq \mathbf{y} \leq \boldsymbol{\beta}$ and $\left|z_{k}\right|<L+1$ for $k=1, \cdots$, n and $\left|z_{0}\right|<2 \frac{\beta_{M}-\alpha_{m}+2 \epsilon}{h}+L+1$ it follows that $\left|D y_{k}\right|$ and $\left|D z_{k}\right|$ are bounded independently of $\delta>0$. It follows that given $\epsilon>0$, there is $\delta>0$ such that for $0<h<\delta$ there is a solution $\mathbf{y}, \mathbf{z}$ of problem (5), (6) and (7) with

$$
\max \{|y(t, \mathbf{y}, \mathbf{z})-y(t)|: 0 \leq t \leq 1\} \leq \epsilon
$$

and

$$
\max \left\{\left|z(t, \mathbf{y}, \mathbf{z})-y^{\prime}(t)\right|: 0 \leq t \leq 1\right\} \leq \epsilon
$$

where $y$ is a solution of (1) and (5),

$$
\begin{aligned}
& y(t, \mathbf{y}, \mathbf{z})=y_{k}+\left(z_{k+1}+z_{k}\right)\left(t-t_{k}\right) / 2, \text { for } t_{k} \leq t \leq t_{k+1}, 0 \leq k \leq n-1 \text { and } \\
& z(t, \mathbf{y}, \mathbf{z})=z_{k}+\left(f_{k+1}+f_{k}\right)\left(t-t_{k}\right) / 2, \text { for } t_{k} \leq t \leq t_{k+1}, 0 \leq k \leq n-1 .
\end{aligned}
$$

Indeed, using an argument similar to that in the proof of (Gained, 1974, Theorem 2.5$)$ it follows that $(y(t, \mathbf{y}, \mathbf{z}), z(t, \mathbf{y}, \mathbf{z}))$ converges uniformly to a solution of (1) and (5) together with (2) and hence to $\left(y, y^{\prime}\right)$ where $y$ is a solution of (1) and (2).

\section{References}

Boyce, W. E. \& DiPrima, R. C. (1976). Elementary Differential Equations and Boundary Value Problems, 3rd Ed., Wiley: New York.

Denny, V. E. \& Landis, R. B. (1972). A New Method for Solving Two-Point Boundary Value Problems Using Optimal Node Distribution, Journal of Computational Physics, 9, 120-137.

Gaines, R. (1974). Difference Equations Associated with Boundary Value Problems for Second Order Nonlinear Ordinary Differential Equations, SIAM J. Numer. Anal., 11 , 411-434.

Henderson, J. \& Thompson, H. B. (2001). Difference Equations Associated with Fully Nonlinear Boundary Value Problems for Second Order Ordinary Differential Equations, Journal of Difference Equations and Applications, , 297-321.

Kelley, W.G. \& Peterson, A. C. (1991). Difference Equations, An Introduction with Applications, Academic Press, Boston.

Mohamed, M. (2007). A PhD Theses, Existence of Solutions to Continuous and Discrete Boundary Value Problems for Systems of First-Order Equations, The University of Queensland, Australia.

Russell, R. D. (1977). Comparison of collocation and finite differences for two-point boundary value problems, Siam J. Numer. Anal. Vol. 14, No.1.

Thompson, H. B. (1996). Second Order Ordinary Differential Equations with Fully Nonlinear Two Point Boundary Conditions, Pacific J. Math., 172, 255-277. 